\title{
Ship-Suspended Acoustical Transmitter Position Estimation and Motion Compensation
}

\author{
Rex K. Andrew, Member, IEEE, Michael R. Zarnetske, Bruce M. Howe, Member, IEEE, and James A. Mercer
}

\begin{abstract}
An acoustical transmitter was suspended at multiple depths to $800 \mathrm{~m}$ from the research vessel $R / V$ Melville at several stations in the North Pacific in 2004. The 3-D position of the transmitter varied with time due to ship motion and subsurface currents. The transmitter 3-D position and velocity were subsequently estimated using a cable dynamics model forced by ship position, as measured by high-precision global positioning system (GPS), and subsurface currents, as measured by the onboard acoustical Doppler current profiler. These estimated positions and velocities varied in the horizontal up to $\pm 10 \mathrm{~m}$ from the station "center" position, and $\pm 0.5 \mathrm{~m} / \mathrm{s}$ from zero, respectively. Auxiliary measurements indicate that these estimates were accurate along either horizontal coordinate to better than $\pm 2 \mathrm{~m}$ and $\pm 0.05 \mathrm{~m} / \mathrm{s}$, respectively. Transmitter motion dilates the apparent time base of the radiated signal, producing time-varying Doppler effects. Simulation and analysis are used to determine when the induced Doppler effect is significant, and a technique is presented that "de-dopplerizes" a received signal for arbitrary interplatform motion. One example, involving the transmitter motion solutions determined here, shows that the transmitter motion induces a root mean square (RMS) variability of roughly $\lambda / 9$ for a $75-\mathrm{Hz}$ ranging signal on time scales of several minutes: a 41-point de-dopplerizing filter reduced this to $\lambda / 121$.
\end{abstract}

Index Terms-Acoustic tomography, acoustical tracking, Doppler, low-frequency propagation, signal coherence, underwater acoustics.

\section{INTRODUCTION}

A traditional acoustical technique for probing and sampling a water mass involves suspending an acoustical transmitter from a ship and sending signals to a distant underwater receiver. The first recorded application of this technique occurred in 1826, when Colladon and Sturm conducted their celebrated underwater sound propagation experiments in Lake Geneva [1]. One commonly begins the mathematical description of such an experiment with a statement like "Let the source

Manuscript received August 14, 2007; revised January 11, 2010; accepted September 02, 2010. Date of publication October 07, 2010; date of current version November 30, 2010. This work was supported by the U.S. Office of Naval Research code 321OA under Grant N00014-03-1-0181. The work of M. R. Zarnetske was supported by the U.S. Office of Naval Research Special Research Award in Ocean Acoustics for Graduate Trainees N00014-04-1-0416, with additional partial funding from the Applied Physics Laboratory and the School of Oceanography, University of Washington, Seattle.

Associate Editor: C. de Moustier.

R. K. Andrew and J. A. Mercer are with the Applied Physics Laboratory, University of Washington, Seattle, WA 98105 USA (e-mail: randrew @ apl.washington.edu)

B. M. Howe is with the Department of Ocean and Resources Engineering, University of Hawaii at Manoa, Honolulu, HI 96822 USA.

M. R. Zarnetske is with the Sensors and Sonar Systems Department, Naval Undersea Warfare Center, Division Newport, Code 1512, Newport, RI 02841 USA.

Digital Object Identifier 10.1109/JOE.2010.2077750 be at $\mathbf{r}_{T} \ldots$ " However, due to the ever-changing interplay of waves, wind, currents, and other mechanical forces, a more accurate model should denote the position as $\mathbf{r}_{T}(t)$, i.e., time varying. This distinction may be important in long-range ocean acoustical propagation problems, where the absolute positions of the transmitter and the receiver are required, and long integration times are common.

The estimation of a time-varying 3-D position or trajectory is known as the tracking problem. Since seawater is vastly more transparent to sound than to electromagnetic waves, the underwater tracking problem typically involves tracking instrumentation consisting of arrays of acoustical sensors (eitherhydrophones or transponders). The literature has traditionally classified these tracking arrays by size. Following [2], short-baseline arrays have sensors at separation distances of less than roughly $20 \mathrm{~m}$, making it practical to mount the entire array on one platform. Long-baseline arrays have much larger intersensor separations, and typically require a separate platform for each sensor. Long-baseline arrays provide better accuracy over a larger area (or volume), but are much more time consuming to deploy, operate, and recover. Long-baseline arrays are common for long-duration, moored deployments [3] but less practical for short-duration ship-suspended deployments, particularly in the deep ocean where laying and surveying in a tracking array on the ocean floor requires extra time.

The literature provides some guidance for tracking accuracy. In ocean acoustical tomography [3], errors are not to exceed $1 \mathrm{~ms}$, which, if attributed solely to range error, corresponds to about 1.5 $\mathrm{m}$. An alternate measure [4] specifies one-quarter of the wavelength of the ranging signal, or $\lambda / 4$. These are generally for applications using travel time to sense oceanic parameters. Coherent processing, e.g., beamforming or long-time integration, has more strict requirements: a standard array sensor tolerance, for example, is one-tenth of a wavelength, or $\lambda / 10$ [5].

Techniques have been developed for establishing a surfacebased tracking system for "moving ship tomography"[6], primarily in conjunction with ship-suspended hydrophone arrays. However, of the numerous long-range acoustical experiments performed, only a few have used a ship-suspended transmitter. The most notable experiments were as follows.

1) The Heard Island Feasibility Test [7] in which a transducer array suspended from the $R / V$ Cory Chouest sent signals up to $10 \mathrm{Mm}$ around the world. During transmission, the ship attempted to maintain a slow but steady heading and way in heavy seas (essentially towing the array). The array was not tracked separately per se: postcruise inversion of acoustical travel times revealed "surges" of the array off the mean track, which corresponded closely, via the ship's global positioning system (GPS) record, to the deviations 


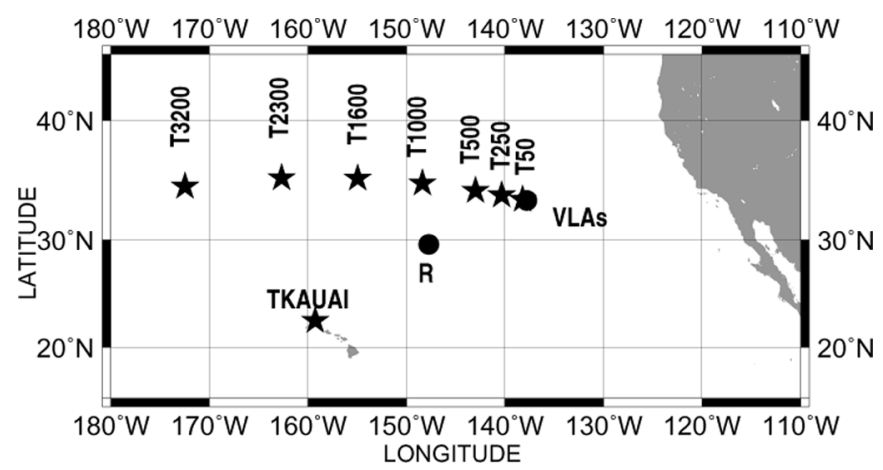

Fig. 1. LOAPEX geometry. The stars designate transmitter deployment stations and the black circles designate receiver systems. All stations (except TKAUAI) were located along the geodesic between T3200 and the VLAs.

of the ship from its own mean track due to wind and waves [8].

2) The Acoustic Thermometry of Ocean/Climate (ATOC) Engineering Test [9] in which an ATOC transmitter was deployed for a week from the R/P FLIP which had been placed in a trimoor. A long-baseline tracking array was placed on the seafloor around $R / P F L I P$ and an acoustical interrogator on the transmitter package enabled the estimation of transmitter position with an accuracy of about 1-m root mean square (RMS). Given this accuracy (the receivers were tracked with similar accuracy), it was possible to determine that the time of flight from transmitter to receivers over $3250 \mathrm{~km}$ depended significantly not only on the time-varying transmitter-receiver separation, but also on the intervening time-varying barotropic tidal currents.

3) The Alternate Source Test [10] in which an HLF-6 transmitter radiating simultaneous coherent $28-$ and $84-\mathrm{Hz}$ signals was deployed from the $M / V$ Independence. This vessel used dynamic positioning (DP) based on its own P-code GPS to maintain station within a nominal $10-\mathrm{m}$ radius circle. The antenna of a second (differential) GPS receiver was mounted at the sheave directly above the top of the suspension cable, and the position of the antenna was used as an approximation for the transmitter position. However, there were no independent measurements of the transmitter position, so the accuracy of this approximation remained unknown.

The primary subject of this paper is a novel approach for estimating the position of a device deployed over the side of a ship to depths of $800 \mathrm{~m}$ with an accuracy of better than $2 \mathrm{~m}$. For low-frequency experiments, in which a 2-m error can correspond to $\lambda / 10$ or less, this accuracy may be sufficient for coherent processing applications. The technique, used for the first time during a 2004 experiment (described in Section II), does not require a tracking array. Contrary to the Alternate Source Test, several auxiliary instruments and an expendable, partial long-baseline acoustical tracking array were deployed to corroborate the position solutions. Results presented below confirm the accuracy of the technique.

The second half of the paper addresses the consequences of transmitter motion, using as an example the estimated trajectory from the 2004 experiment. Measurements suggest that the true transmitter position fluctuated about the design position on horizontal scales as large as a half-wavelength, and over time scales comparable to the duration of the ranging signal. A simulation demonstrates for the particular case of the 2004 experiment waveform and a common coherent processor that, as expected, this motion introduces considerable processor performance degradation. A simple "de-dopplerization" procedure is then presented that substantially mitigates influence of this motion.

Therefore, this paper is organized as follows. Section II briefly reviews key features of the 2004 experiment. Section III presents the position estimation technique and discusses its accuracy. Section IV studies the consequences of transmitter motion. Section V develops and applies the de-dopplerization algorithm. Section VI summarizes the principal results.

\section{THE 2004 LONG-RANGE OCEAN ACOUSTIC PROPAGATION EXPERIMENT}

The tracking approach described here was used in the Long-range Ocean Acoustic Propagation EXperiment (LOAPEX), which was conducted in the North Pacific in September-October 2004. LOAPEX was designed to provide range-varying acoustical data for understanding the basic physics of low-frequency, long-range, broadband propagation [11], [12]. An ATOC HX554 low-frequency electroacoustical transducer [13] was suspended from the $R / V$ Melville at multiple stations along a predominantly east-west geodesic track north of the Hawaiian islands, as shown in Fig. 1. At each station, the transmitter was deployed at a few selected depths down to $800 \mathrm{~m}$ for periods from hours to days. The acoustical signals involved carrier frequencies of about $75 \mathrm{~Hz}$, and therefore wavelengths of about $20 \mathrm{~m}$. The transmissions were measured by a variety of instruments throughout the North Pacific, including a network of bottom-mounted receivers cabled to shore (one of which-receiver $\mathbf{R}$-is shown in Fig. 1), and several vertical hydrophone line arrays (VLAs).

The $R / V$ Melville possesses a DP system which uses two Z-drive propellers and a bow thruster to maintain its reference point at a fixed "design" geolocation. Wind, waves, and surface currents continuously forced the ship "off position," and therefore the DP system continuously applied corrections. As a result, the reference point executed a random trajectory around and through the design geolocation point; the transmitter swung back and forth in response.

The position of the reference point aboard the $R / V$ Melville relative to the design geolocation is easily measured, e.g., via GPS: however, the desired quantity is the transmitter position. Preliminary calculations indicated that the transmitter position could deviate from its design position by more than $\lambda / 10$, and therefore these deviations could not simply be ignored. For the deployment depths under consideration here (to $800 \mathrm{~m}$ ), a shortbaseline tracking array would have been inadequate, and there was neither the time nor resources available to establish a longbaseline system at each station. Consequently, a new approach, presented in Section III, was developed. 


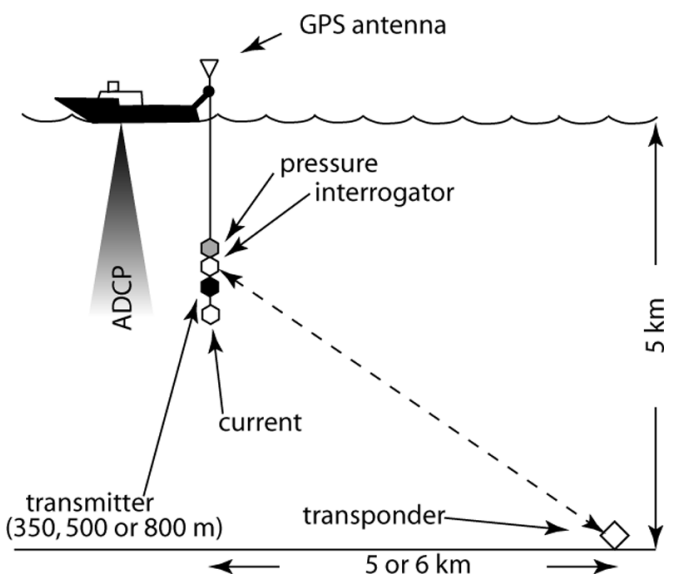

Fig. 2. Transmitter tracking instrumentation. The primary instruments were the GPS (with the antenna directly over the suspension cable) and the ADCP. Auxiliary instruments consisted of a pressure sensor, a current meter, and an acoustical interrogator ranging (dashed line) off an unsurveyed transponder deployed onto the ocean floor. Not to scale.

\section{TRANSMitTER Position ESTIMATION}

This section describes the complete process of position estimation, including 1) the cable model and the primary measurements used to define the forcing functions in the model, 2) auxiliary instrumentation used at each station for additional corroborating measurements, and 3) a summary of the accuracy of the solutions. The primary and auxiliary instrumentations used at each station for transmitter tracking are shown in Fig. 2.

\section{A. A Model-Based Estimator}

The unknown transmitter position was estimated using three components: 1) a dynamical cable model, 2) translations of the cable suspension point from the design geolocation, and 3) subsurface currents. All three components are described below.

1) The Cable Dynamics Model: The program "WHOI Cable" [14] is a time-domain numerical simulation tool for moored and towed oceanographic systems. It was originally formulated for mooring system design, and it allows for the inclusion of typical ocean conditions and mooring instruments and components. The code is based on coupled nonlinear differential equations for 2-D and 3-D static and dynamic problems [15], [16] and solves for the 3-D shape of an underwater cable. The forces on each differential element of cable are hydrostatic (i.e., gravity and buoyancy), hydrodynamic (horizontal and vertical drag due to the relative fluid velocity at the element, added mass due to the acceleration of the element relative to that of the local fluid, and a dynamic Archimedes force due to a pressure gradient that arises from a time rate of change of the local fluid velocity) and the change in tension from one end of the element to the other. The time rate of change of the angular momentum of the element is controlled by the tensions, rotational inertia, shear, and element bending. Material nonlinearities may be included. In addition, each element supports elastic deformation, enabling the code to model cable elongation under tension. This introduces additional equations of compatibility and continuity to relate strain tensors to displacements and enforce continuity of solutions between elements along the length of the cable.
TABLE I

INPUT PARAMETERS TO THE WHOI CABLE CODE

\begin{tabular}{|c|c|}
\hline \multicolumn{2}{|c|}{ SHIP/PLATFORM } \\
\hline $\begin{array}{c}\text { GPS position } \\
\text { ADCP profiles }\end{array}$ & $\begin{array}{c}\text { position time series } \\
\text { velocity time series }\end{array}$ \\
\hline \multicolumn{2}{|c|}{ TRANSDUCER (right cylinder) } \\
\hline diameter & $1.1 \mathrm{~m}$ \\
height \\
buoyancy \\
mass \\
horizontal drag coefficient
\end{tabular}

The system of equations is solved numerically by discretizing the continuous forms of the governing equations using spatial finite differences centered on grid points defined by the user. This is known as the generalized- $\alpha$ algorithm, which offers superior accuracy and stability when compared to the box method, trapezoidal rule, or backward differences [16], [17]. The code uses the initial position given by ship position, and the initial current profile to determine a zero position for the transmitter. At each step of the solution, the system of nonlinear equations is solved by an iterative, implicit, adaptive relaxation technique [16], [18, ch. 19]. The initial guess for the solution in the static problem is calculated using a shooting method. For the dynamic solution, the initial guess at each time step is the solution from the previous time step. For each iteration, the equations are solved using a sparse Gaussian elimination algorithm for which the computational effort scales linearly with the number of nodes [19]. The input parameters for the algorithm are shown in Table I.

For this application, the code is used in "towing" mode, wherein one end of the cable is forced by the motion of the ship and the other end is free to respond under the action of the fluid velocity on the cable and attached packages. WHOI Cable has not been used previously to model a ship-deployed transmitter configuration. Therefore, a sensitivity study was performed to determine cable and transmitter drag coefficients. A representative 30-min portion of transmitter position solutions from station T3200 was used because the weather at T3200 involved 


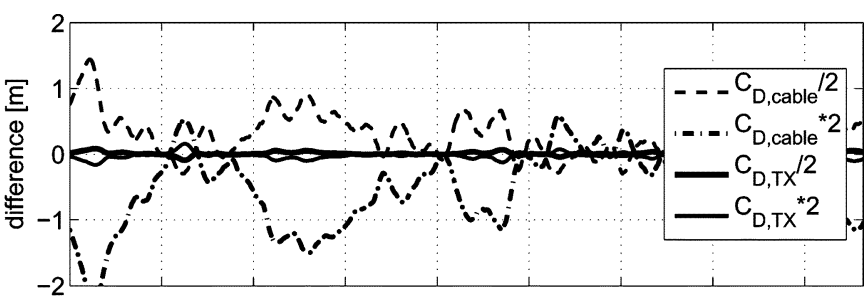

(a)

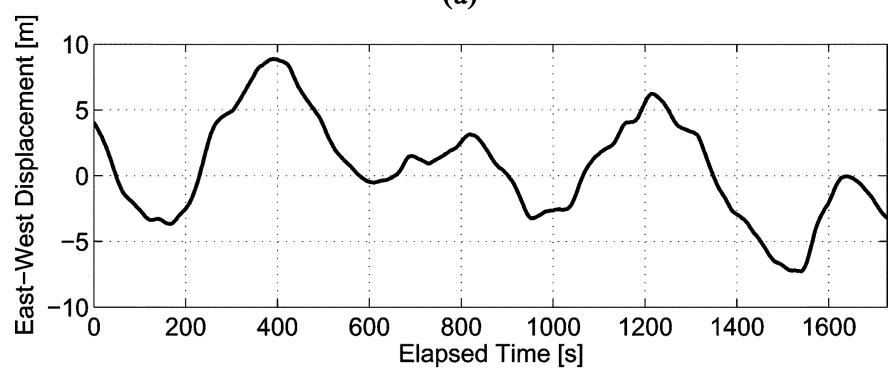

(b)

Fig. 3. Comparison of drag study results. $C_{D \text {, cable }}$ is the cable horizontal drag coefficient with nominal "literature" value 1.5. $C_{D, \mathrm{TX}}$ is the transmitter horizontal drag coefficient with nominal value 0.6. (a) Difference functions for the four alternative parameter values. (b) The solution for the nominal parameter values. The time axis is referenced to October 3, 2004, yearday 277, 13:26:24Z .

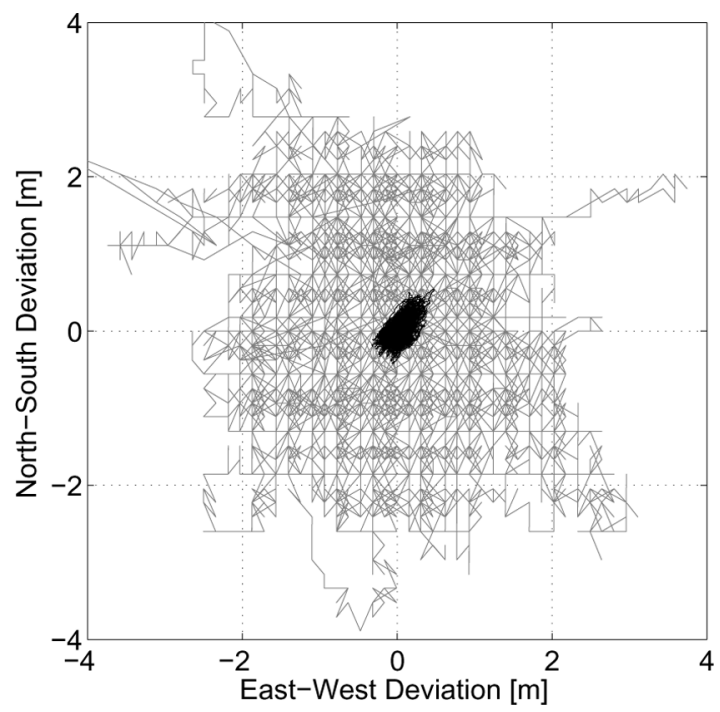

Fig. 4. Comparison of horizontal position solutions, onboard GPS systems. Black: C-Nav. Gray: Furuno. The solutions from the Trimble GPS were similar in character to the Furuno solutions, and are not shown for clarity. Approximately $9.5 \mathrm{~h}$ of data. $R / \mathrm{V}$ Melville moored at the dock and stationary.

some of the roughest seas and highest winds, which caused the most vigorous horizontal forcing at the top of the cable during the experiment. Estimates of the initial drag coefficients were based on similar earlier studies [20]-[22]. Initial values of 1.5 and 0.01 were used for the horizontal and vertical coefficients of the cable, respectively. The transmitter was modeled as a cylinder with an initial horizontal coefficient of 0.6 . The model does not require a vertical coefficient for the transmitter. For the sensitivity study, the horizontal drag coefficients were halved and doubled for both the cable and for the transmitter, in independent trials. Representative difference time series for all trials are shown in Fig. 3. The effect of the transmitter drag coefficient on the positioning of the transmitter was negligible, thus the cable horizontal drag coefficient dominates. The large range of cable drag coefficient values changed the transmitter position by less than $1 \mathrm{~m}$. Therefore, the horizontal and vertical cable coefficients were fixed at the values found in the literature of 1.5 and 0.01 , respectively, and the horizontal transmitter coefficient was set at 0.6 as well.

2) Suspension Point Position: The $(x, y, z)$ origin of the transmitter frame of reference was the design station geolocation; the position of the top of the suspension cable in this frame of reference was measured directly by fixing a GPS antenna on the stern A-frame precisely over the suspension point. ${ }^{1}$

The GPS was a component of the C-Nav system, which provides worldwide dual-frequency corrected GPS coverage. The dual-frequency corrects for ionospheric errors, while data from globally distributed ground stations are downlinked to the shipboard receiver in real time from geostationary satellites to correct for GPS satellite ephemeris errors, GPS clock error, and other atmospheric effects. Position is sampled once per second and the accuracy is specified to be subdecimeter.

In addition to time, latitude, longitude, and altitude, the $\mathrm{C}-\mathrm{Nav}$ system records other parameters that help indicate the quality of the received signals and accuracy of the position estimates. For example, stations T2300 and T3200 were located in the transition region between the coverage zones of two of the geostationary satellites, and the correction downlink receptions were therefore intermittent due to the low declination of those satellites combined with interference from the ship's superstructure.

Measurements made at the pier in San Diego, CA, before the departure for the LOAPEX cruise, confirm the ability of $\mathrm{C}-\mathrm{Nav}$ to provide decimeter accuracy precision measurements with no bias over long periods of time, on the order of a day or two, commensurate with the anticipated duration of transmitter station deployments. The $R / V$ Melville was outfitted with two other GPS systems, a Trimble dual-frequency P-code receiver and a Furuno single-frequency C/A code GP-90 receiver. A pier-side comparison showed the $\mathrm{C}-\mathrm{Nav}$ to be superior in precision (Table II and Figs. 4 and 5). The C-Nav data were uniformly smooth and the deviations about the mean position shown in Table II were an order of magnitude lower than for the other two GPS systems. Also, both the P-Code and the C/A Code data showed significant jumps and a wide range of variability. The sinusoidal variation in the C-Nav vertical height corresponds to the tidal signal at San Diego. The tide tables for the harbor predict a $1.12-\mathrm{m}$ peak-peak tide change between a low at 08:30:00Z and a high at 15:28:00Z (during yearday 253). This $1.12-\mathrm{m}$ signal is nearly exactly measured by the C-Nav system (Fig. 5).

3) Subsurface Current Profiles: The $R / V$ Melville was outfitted with an RD Instruments (Poway, CA) Ocean Surveyor $75-\mathrm{kHz}$ acoustical Doppler current profiler (ADCP). This instrument averaged acoustical backscatter data over depth cells and time ensembles. Spatially, depth cells are windows along the entire profiling depth that are all individually averaged.

\footnotetext{
${ }^{1}$ This contrasts with the $R / V$ Melville's own GPS antennas, which, at that time, measured positions above the bridge. When the antenna is mounted on the A-frame, no further "offset" correction is necessary to yield a measurement referenced to the design geolocation $(x, y, z)$ origin.
} 


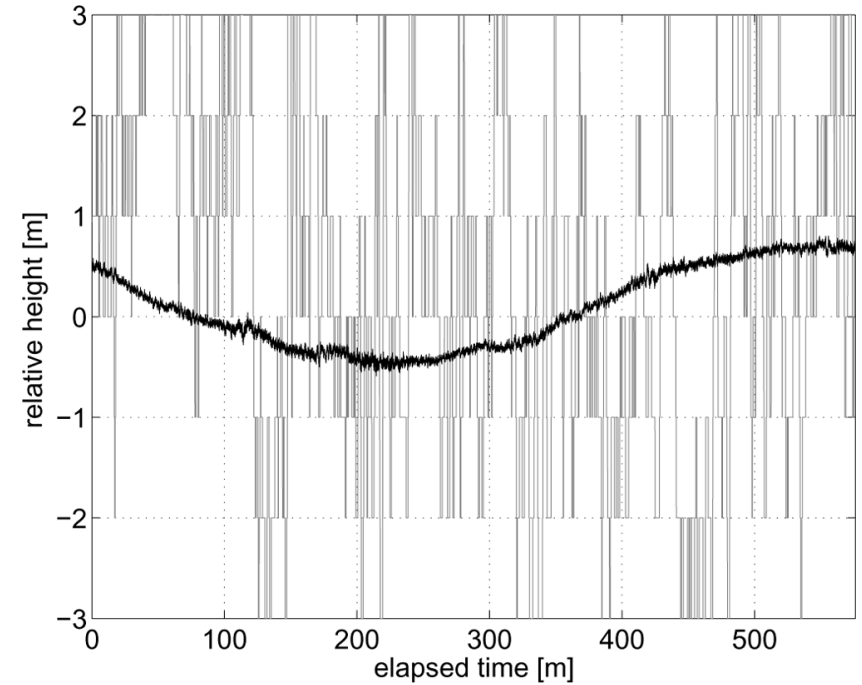

Fig. 5. Comparison of vertical position solutions, onboard GPS systems. Black: C-Nav. Gray: Furuno. The solutions from the Trimble GPS were similar in character to the Furuno solutions. Approximately $9.5 \mathrm{~h}$ of data. $R / V$ Melville moored at the dock, but rising and falling with the tide. The $x$-axis reference is September 9, 2004, yearday 253, 04:48:00Z.

TABLE II

COMPARISON OF POSITION VARIABILITY (RMS/PEAK-TO-PEAK) (IN METERS) FOR THE THREE ONBOARD GPS SYSTEMS, PIER-SIDE ( $R / V$ MELVILLE STATIONARY)

\begin{tabular}{|c|c|c|c|}
\hline \multirow{2}{*}{ orientation } & \multicolumn{3}{|c|}{ instrument } \\
\cline { 2 - 4 } & C-Nav & Trimble & Furuno \\
\hline East/West & $0.11 / 0.86$ & $0.66 / 5.14$ & $0.88 / 9.8$ \\
North/South & $0.12 / 0.97$ & $1.20 / 6.67$ & $1.1 / 8.33$ \\
vertical & $0.40 / 1.39$ & $2.36 / 17.3$ & $2.1 / 16$ \\
\hline
\end{tabular}

This averaging reduces the effects of noisy data. The depth bins used started at $24 \mathrm{~m}$ and continued downward at 16-m intervals to $800 \mathrm{~m}$ total. Temporally, a velocity profile was recorded every minute. These measurements were then combined to create 5-min ensembles. The averaging process reduces the measurement uncertainty and random errors introduced by the positioning systems, producing more consistent absolute velocity averages.

According to the manufacturer, the depth range, with the 16-m depth bin used during the cruise, is $560-700 \mathrm{~m}$ due to the limited number of scatterers at depth. However, in this application, useful data were measured down to $800 \mathrm{~m}$, perhaps because the ship was keeping station, which would reduce flow noise and bubble production around the hull sensor. For velocity accuracy, the manufacturer specifies $\approx 0.5 \mathrm{~cm} / \mathrm{s}$ for the deepest bin using 5-min averaging. To create the absolute velocity components of the current beneath the ship, the movement of the ship was removed using the P-Code GPS signal from the ship's Trimble receiver.

To quantify the effect the local currents have on transmitter displacements, the depth-averaged current velocity was determined for station T250 when the transmitter was at 800-m depth (Fig. 6). The currents are clearly small. Over the time series

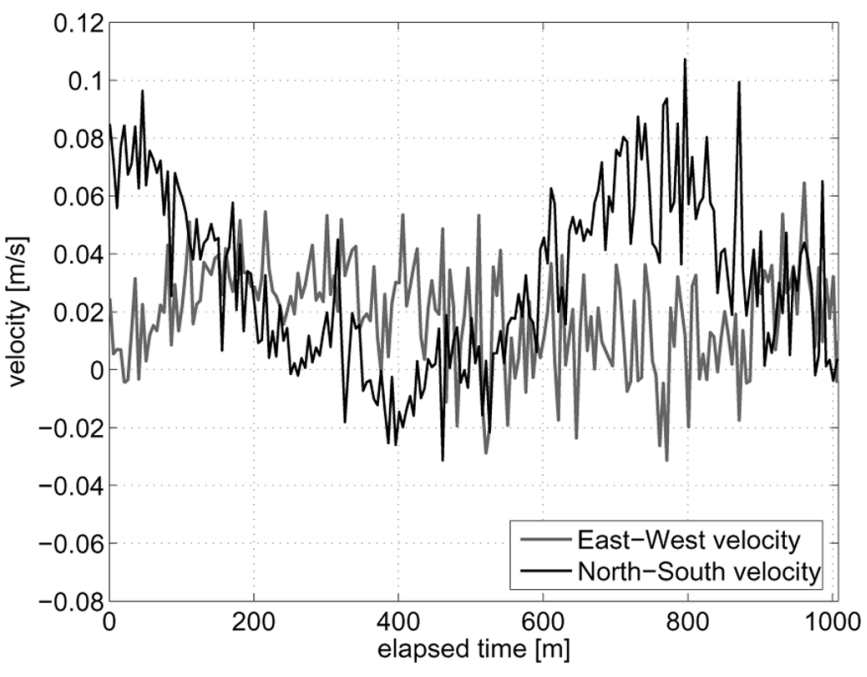

Fig. 6. Depth-averaged current during transmitter deployment to $800 \mathrm{~m}$ at station T250. The $x$-axis reference is September 16, 2004, yearday 260, 19:12:00Z.

shown, the mean current has a magnitude of $3.9 \mathrm{~cm} / \mathrm{s}$ and a mean bearing of $62.4^{\circ}$. This mean current caused a $2-3-\mathrm{m}$ shift of the transmitter position over the duration of the deployment in the general northeast direction. Current magnitudes similar to this were recorded at the other stations.

\section{B. Auxiliary Instrumentation}

Additional instrumentation was used at each station to either augment or corroborate the primary measurements (see Fig. 2). These were as follows.

- An InterOcean Systems (San Diego, CA) S4 current meter, suspended about $10 \mathrm{~m}$ beneath the transmitter, to measure the current velocity at the transmitter depth. This sensor measures the voltage resulting from the motion of a conductor (i.e., seawater) through a magnetic field generated by the instrument. Two orthogonal pairs of electrodes and an internal flux gate compass provide the current vector. It sampled once every $30 \mathrm{~s}$.

- A Sea-Bird Electronics (Bellevue, WA) SBE 37-SM MicroCAT pressure sensor, affixed to the deployment cable about $25 \mathrm{~m}$ above the transmitter, to measure hydrostatic pressure, from which the depth of the transmitter can be derived. The MicroCAT also measured temperature, but the temperature time series was not used here.

- An acoustical interrogator, attached to the deployment cable about $20 \mathrm{~m}$ above the transmitter. This operated at each station in conjunction with a Benthos (North Falmouth, MA) transponder to construct a partial long-baseline tracking array (described more fully below). The interrogation period was $6 \mathrm{~s}$ for all stations, except at station T3200, where the period was $3 \mathrm{~s}$.

It was originally thought that the data from these instruments would be used to tune the parameters of the cable, but these data did not have high enough quality to improve upon the cable model estimates. However, these auxiliary data are sufficient to corroborate the cable model solutions. These comparisons are presented in Section IV. 


\section{Corroborating Measurements}

1) Horizontal 1-D Displacement: A partial acoustical tracking configuration was constructed at each station by dropping a transponder approximately $5 \mathrm{~km}$ eastward of each station, thereby placing it on the local tangent to the T3200-VLA geodesic LOAPEX track. Due to constraints on available ship time, the final position of the transponder on the seafloor was not surveyed: its position was approximated using the known surface drop position and the depth of the water as measured by the ship's echosounder. The transponder was not subsequently recovered.

The relative position along the geodesic to the VLA is determined from

$$
\delta x=\frac{-c_{0}}{\cos \theta_{0}} \delta t
$$

where $c_{0}$ is the average sound speed $(1480 \mathrm{~m} / \mathrm{s}), \theta_{0}$ is the ray angle from the transmitter to the transponder using the approximate (straight-line) geometry, and $2 \delta t=\left(T T-T T_{0}\right)$ is the difference between the measured $(T T)$ and average round trip travel times $\left(T T_{0}\right)$.

This was not a complete tracking solution because it only tracked the transmitter along the 1-D bearing back to the transponder, and, in addition, did not provide absolute positioning because the transponder position was unknown. However, the scheme did provide an independent measure of the relative transmitter position along a single bearing, which can be compared to the projection of the model solutions along the same bearing.

The interrogator/transponder time series was highly variable due to low signal-to-noise ratio (SNR). To reduce the variability of the time series, the data was median filtered using a running 30-s window. Also, a known $66 \mathrm{~ms} / \mathrm{h}$ increase, or time shortening, internal clock error was removed. The estimated $\delta x$ and the model solution for $30 \mathrm{~min}$ of deployment time at station T250 are shown in Fig. 7. The interrogator/transponder time series very nearly overlays the transmitter position, except for the beginning of the data set. The difference between the interrogator/transponder time series and the transmitter position time series in the first $800 \mathrm{~s}$ is likely caused by the limited number of clean samples for the interrogator/transponder travel time. For instance, a higher correlation results if the five points lying between 430 and $700 \mathrm{~s}$ and below $-3.5 \mathrm{~m}$ are eliminated. Another feature of the curve shown in Fig. 7 is the approximate 6-min oscillation. The ship was dynamically positioned for all stations and the reaction of this system to correct the position of the ship nominally followed a 5-10-min cycle.

A summary of comparisons between the model and the interrogator/transponder measures is shown in Table III. The correlation coefficient $\rho_{x y}$ remained acceptably high with a value of 0.84 averaged over the values at each station. The RMS difference between the model estimates and the interrogator time series averaged $1.5 \mathrm{~m}$ over all stations; this is less than $\lambda / 10$ at the transmission signal center frequency of $75 \mathrm{~Hz}$. By comparison, the maximum deviations of the transmitter position from its design position over all stations, using the model estimates, were roughly $\pm 10 \mathrm{~m}$. Positioning accuracy is clearly improved

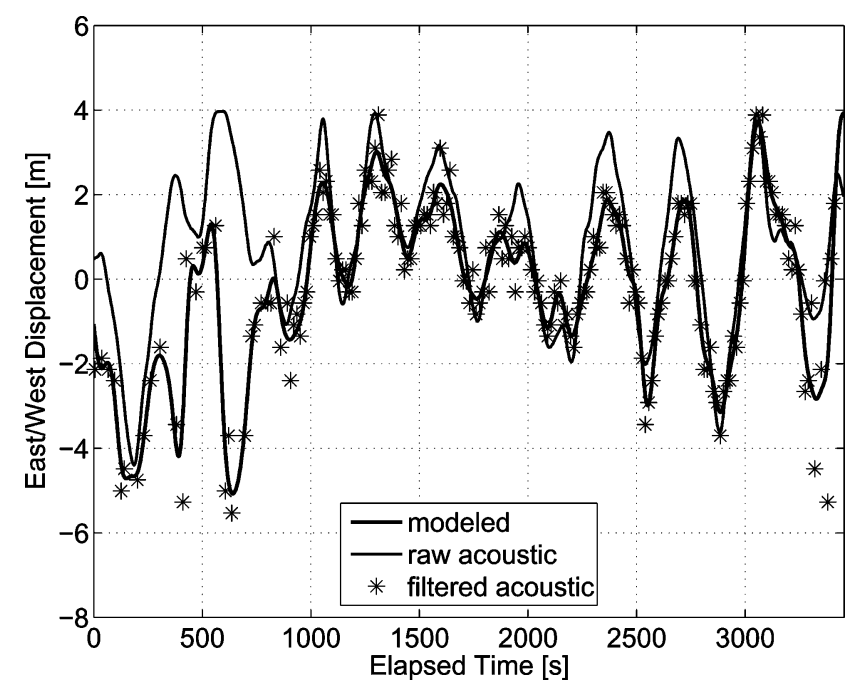

Fig. 7. East/west displacements as measured by the acoustical tracking system, compared to the model solutions. Station T250, transmitter depth $800 \mathrm{~m}$. The $x$-axis reference is September 16, 2004, yearday 260, 23:02:24Z.

TABLE III

Comparison Between the TransmitTer Model (EAST/WeSt) Position USING AND THE 1-D ACOUSTICAL TRACKING SOLUTION (NOMINALlY EAST/WEST). VALUES AVERAGED OVER THE ENTIRE DEPLOYMENT FOR EACH DEPTH FOR EACH STATION. DEPTH IS IN UNITS OF METERS, AND $\Delta x$ IS IN UNITS OF RMS METERS

\begin{tabular}{|c|c|c|c|}
\hline station & depth & $\rho_{\mathrm{xy}}$ & $\Delta \mathrm{x}$ \\
\hline T250 & 350 & 0.82 & 1.5 \\
& 800 & 0.77 & 1.7 \\
\hline T500 & 350 & 0.93 & 1.6 \\
& 800 & 0.96 & 1.4 \\
\hline T1600 & 350 & 0.82 & 0.6 \\
\hline T3200 & 350 & 0.85 & 1.5 \\
& 500 & 0.71 & 2.2 \\
\hline
\end{tabular}

by assuming the model-estimated trajectory over a stationary, fixed position.

2) Vertical Displacement: Fig. 8 shows an excerpt of the comparison of the pressure sensor depth overlaid with the transmitter model position solution, and the vertical (relative) position of the ship, as recorded by the C-Nav GPS system. These comparisons revealed that the amplitude of the pressure sensor depth variation was approximately $60 \%$ that of the antenna. This discrepancy cannot be attributed to dynamic stretch in the cable: the model incorporates cable stretching, based on the effective Young's modulus of the cable, and the effect of the stretch of the cable on the transmitter position is estimated as negligible. A more plausible explanation notes that the sensor acquired 6-s averages logged every $15 \mathrm{~s}$, thus smoothing the time series by averaging, but also aliasing the time series by only taking one sample every $15 \mathrm{~s}$.

3) Horizontal Velocities: The current meter data for each station show similar character and statistics as those estimated by the model. Fig. 9 shows both components of the current measured during deployment at station T250. The spikes and steps 


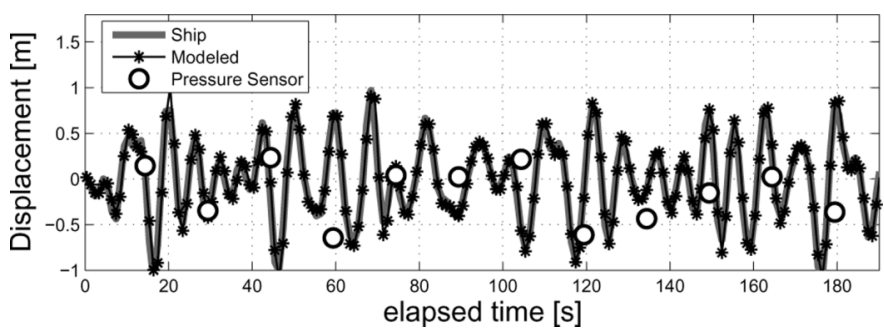

Fig. 8. Displacements about the mean for the measured (via the pressure sensor) and modeled vertical transmitter position overlaid on the ship's vertical displacement (as measured by the GPS) for the 800-m deployment at station T250. The modeled and GPS solutions are virtually indistinguishable. The $x$-axis reference is September 16, 2004, yearday 260, 23:22:33Z.
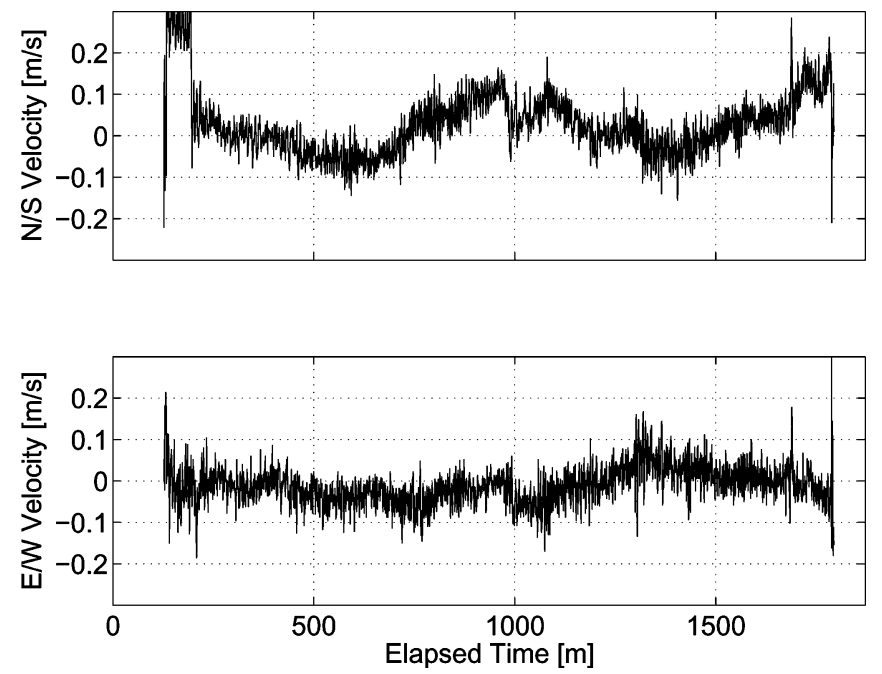

Fig. 9. Current meter measurements at station T250. The $x$-axis reference is September 16, 2004, yearday 260, 14:24:00Z.

at the beginning and the end of the time series are the result of deployment and recovery transients.

The current meter record minus the absolute velocity of the water measured by the ADCP at the transmitter depth yields the absolute velocity of the transmitter. Fig. 10 shows an expanded comparison of the transmitter model velocity and this absolute transmitter velocity (from the current meter/ADCP difference) from station T250 at 800-m depth. The modeled and measured low-frequency trends in the east/west and north/south transmitter velocities compare well.

Table IV shows a quantitative comparison for all stations, based on the estimated velocity, and the current meter/ADCP difference. Ideally, a higher correlation coefficient, and a shorter lag time should be expected, but one must consider that the ADCP is sampling deeper than its specified depth $(<700 \mathrm{~m})$ and the mismatch in sampling frequencies between the current meter (30 s) and the ADCP (5-min averages). To determine the absolute transmitter velocity, the ADCP 5-min bin averages to $800 \mathrm{~m}$ were subtracted from each of the current meter readings occurring within that $5 \mathrm{~min}$. At station T3200, the correlation coefficients are small and the RMS difference is large. The higher sea state present at this station, combined with the sample frequency difference between the current meter and the ADCP, contributed to the poor comparison. Fig. 11 shows a graphical correlation
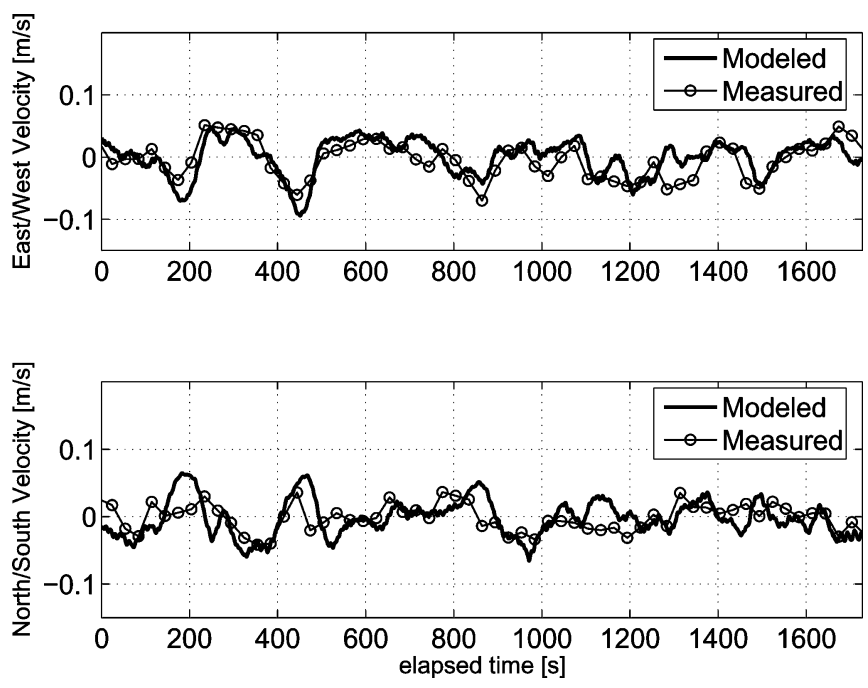

Fig. 10. Expanded view of the comparison between the modeled horizontal transmitter velocity and the current meter/ADCP difference (labeled "measured") at station T250. The $x$-axis reference is September 16, 2004, yearday 260, 22:33:36Z.

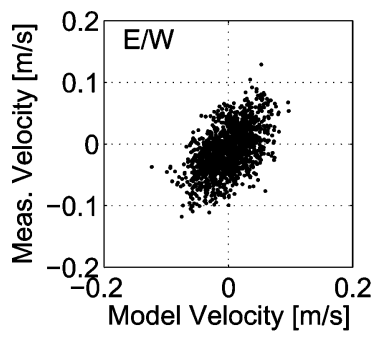

(a)

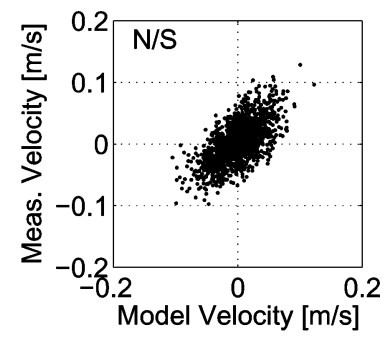

(b)
Fig. 11. Scatter plot between modeled and measured (current meter/ADCP) transmitter velocities: (a) east/west component; (b) north/south component.

between the current meter/ADCP difference and the transmitter model velocity at station T250.

To summarize, over all stations, the average RMS difference was $0.052 \mathrm{~m} / \mathrm{s}$, while the average RMS transmitter velocity from the cable model was $0.54 \mathrm{~m} / \mathrm{s}$, and the average correlation was 0.5 .

\section{Tracking Estimation Summary}

In summary, the independent measurements of transmitter motion compared well to the cable-model estimated motion. The average correlation coefficient between the modeled and measured horizontal position was 0.84 , with an average error of $15 \%$ of the maximum displacements. The average correlation coefficient between the modeled and measured horizontal velocity was 0.5 , with better correlations in milder seas and poorer correlations in rougher seas. The RMS amplitude of the measured vertical displacement was only about $60 \%$ that of the modeled vertical displacement, but the validity of this comparison is suspect because the depth sensor data acquisition protocol was inappropriate: future tests should involve a sampling rate high enough to resolve principal wind-wave-induced heave frequencies. A complete listing of performance comparison statistics [23] indicates that the cable model, using only forcing from the ADCP current profile and the GPS cable-top position, models 
TABLE IV

MEASURED AND ESTIMATED TRANSMITTER VELOCITY COMPARISON. DEPTH IS IN UNITS OF METERS, $\Delta T$ IS THE DURATION IN HOURS OF THE DATA TIME SERIES AT THAT DEPTH, AND RMS $\Delta V$ IS THE RMS VELOCITY DIFFERENCE IN UNITS OF METERS PER SECOND

\begin{tabular}{|c|c|c|c|c|c|}
\hline station & depth & $\Delta \mathrm{T}$ & direction & $\rho_{\mathrm{xy}}$ & RMS $\Delta v$ \\
\hline \multirow[t]{4}{*}{$\mathrm{T} 50$} & 350 & 11.68 & $\mathrm{E} / \mathrm{W}$ & 0.67 & 0.04 \\
\hline & & & $\mathrm{N} / \mathrm{S}$ & 0.78 & 0.04 \\
\hline & 800 & 14.63 & $\mathrm{E} / \mathrm{W}$ & 0.27 & 0.07 \\
\hline & & & $\mathrm{N} / \mathrm{S}$ & 0.32 & 0.07 \\
\hline \multirow[t]{4}{*}{$\mathrm{T} 250$} & 350 & 9.40 & $\mathrm{E} / \mathrm{W}$ & 0.56 & 0.04 \\
\hline & & & $\mathrm{N} / \mathrm{S}$ & 0.45 & 0.05 \\
\hline & 800 & 14.60 & $\mathrm{E} / \mathrm{W}$ & 0.46 & 0.04 \\
\hline & & & $\mathrm{N} / \mathrm{S}$ & 0.56 & 0.03 \\
\hline \multirow[t]{4}{*}{ T500 } & 350 & 15.83 & $\mathrm{E} / \mathrm{W}$ & 0.57 & 0.04 \\
\hline & & & $\mathrm{N} / \mathrm{S}$ & 0.69 & 0.03 \\
\hline & 800 & 20.00 & $\mathrm{E} / \mathrm{W}$ & 0.43 & 0.04 \\
\hline & & & $\mathrm{N} / \mathrm{S}$ & 0.59 & 0.04 \\
\hline \multirow[t]{4}{*}{$\mathrm{T} 1000$} & 350 & 35.61 & $\mathrm{E} / \mathrm{W}$ & 0.65 & 0.03 \\
\hline & & & $\mathrm{N} / \mathrm{S}$ & 0.63 & 0.04 \\
\hline & 800 & 7.44 & $\mathrm{E} / \mathrm{W}$ & 0.49 & 0.04 \\
\hline & & & $\mathrm{N} / \mathrm{S}$ & 0.47 & 0.04 \\
\hline \multirow[t]{2}{*}{ T1600 } & 350 & 25.87 & $\mathrm{E} / \mathrm{W}$ & 0.45 & 0.05 \\
\hline & & & $\mathrm{N} / \mathrm{S}$ & 0.64 & 0.04 \\
\hline \multirow[t]{4}{*}{ T2300 } & 350 & 13.41 & $\mathrm{E} / \mathrm{W}$ & 0.35 & 0.07 \\
\hline & & & $\mathrm{N} / \mathrm{S}$ & 0.43 & 0.06 \\
\hline & 500 & 14.80 & $\mathrm{E} / \mathrm{W}$ & 0.36 & 0.06 \\
\hline & & & $\mathrm{N} / \mathrm{S}$ & 0.44 & 0.06 \\
\hline \multirow[t]{4}{*}{ T3200 } & 350 & 15.49 & $\mathrm{E} / \mathrm{W}$ & 0.60 & 0.04 \\
\hline & & & $\mathrm{N} / \mathrm{S}$ & 0.51 & 0.06 \\
\hline & 500 & 15.92 & $E / W$ & 0.25 & 0.09 \\
\hline & & & $\mathrm{N} / \mathrm{S}$ & 0.17 & 0.12 \\
\hline
\end{tabular}

the dynamics of an underwater package with enough accuracy to yield an estimated trajectory with an RMS error of about 1-2 $\mathrm{m}$ at depths up to $800 \mathrm{~m}$.

\section{DOPPLER EFFECTS}

Although the $R / V$ Melville used its DP capability in an attempt to maintain a fixed position at each station, the transmitter swung freely about its design position during each deployment. The time-varying Doppler "shift" induced by this motion is not a simple frequency shift, as would be the case in a constant velocity scenario, but rather a time-varying time-base transformation. Therefore, the effects of transmitter motion are difficult to intuit from a simple analysis. The methodology used here, instead, is to synthesize an exact "dopplerized" signal, and then investigate the effects on signal processing by direct application of the processing algorithms on the synthetic data.

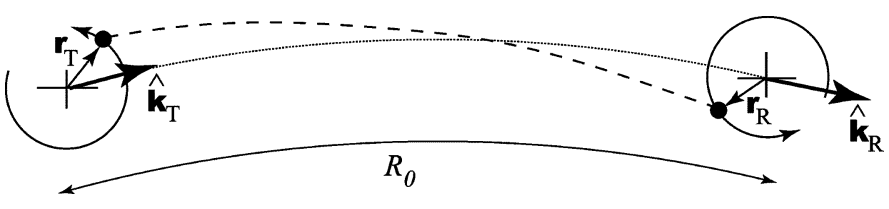

Fig. 12. Geometric definitions for the moving transmitter/moving receiver problem. The problem context involves considerable distances through the Earth's oceans, and therefore the Fermat path of signal propagation between the transmitter site and the receiver site, which is the acoustical "line-of-sight" path, is represented as a curve. The actual path (dashed line) is approximately parallel to the path joining origins (solid line) for long-range problems. The transmitter and receiver "revolving orbits" are simple renditions representing arbitrarily complicated motion confined to a small neighborhood around the origins of the transmitter/receiver coordinate systems.

\section{A. Forward and Inverse Time-Base Mapping}

A schematic diagram of the model geometry is shown in Fig. 12. The transmitter is located at $\mathbf{r}_{T}(t)$ in a local coordinate system, and the receiver at $\mathbf{r}_{R}(t)$ in its own local coordinate system. The $y$-axes of these coordinate systems are aligned along local north/south meridians. The acoustical "line-of-sight" line from transmitter origin to receiver origin is effectively a straight line for short distances, but for long-range propagation problems (such as LOAPEX) this line is a curved geodesic track. In either case, this line has arc length $R_{0}$. The vector at the transmitter along the line of sight is $\hat{\mathbf{k}}_{T}$ and at the receiver is $\hat{\mathbf{k}}_{R}$ : neither are functions of time.

The speed of sound in the ocean is a function of position and time, and fluctuates over a wide range of time scales. In long-range propagation, most of the variability in path-integrated travel time is due to large-scale oceanographic processes (such as tides, mesoscale eddies, or seasonal climatic shifts) with time scales much larger than the typical scales of platform motion (which, for the transmitter in LOAPEX, was tens of seconds). With respect to these processes, it is adequate to postulate a simple model of signal propagation involving a single sound speed $c_{0} .^{2}$

Let $t$ be the time on the transmitter platform. It is useful to imagine the transmitter emitting a pulse at $t$. The corresponding time on the receiver (i.e., when the pulse arrives) is [24]

$$
\mathcal{T}(t) \equiv t^{\prime} \approx t+\frac{\left(R_{0}+\mathbf{r}_{R}\left(t^{\prime}\right) \cdot \hat{\mathbf{k}}_{R}-\mathbf{r}_{T}(t) \cdot \hat{\mathbf{k}}_{T}\right)}{c_{0}} .
$$

That is, a pulse emitted from the transmitter at time $t$, when the transmitter is at position $\mathbf{r}_{T}(t)$, arrives at the receiver at time $t^{\prime}$, when the receiver is at position $\mathbf{r}_{R}\left(t^{\prime}\right)$. For long-range propagation, (2) is accurate to first order in either $\left|\mathbf{r}_{T}\right| / R_{0}$ or $\left|\mathbf{r}_{R}\right| / R_{0}$, both of which are assumed to be much less than unity. The mapping $\mathcal{T}(t)$ is one-to-one and monotonic, and therefore admits an inverse mapping

$$
t=\mathcal{T}^{-1}\left(t^{\prime}\right)
$$

Note that if the receiver is stationary (i.e., with a time-invariant position) the forward map can be computed explicitly. Likewise, if the transmitter is stationary but the receiver

\footnotetext{
${ }^{2}$ Internal waves are the dominant source of sound-speed variability on the time scales of platform motion, and therefore impose a fundamental limit on the applicability of this analysis. The nature of this limit is a topic of current research.
} 
moving, the inverse map can be computed explicitly. However, in the general case in which both platforms are moving, neither the forward nor inverse map can be solved explicitly; the general case nonetheless can be cast as straightforward 1-D rooting problems and solved numerically, using e.g., the van Wijngaarden-Dekker-Brent algorithm [18, pp. 359-362].

\section{B. Simulated Doppler-Induced Algorithm Performance Degradation}

Given the mapping $\mathcal{T}(t)$, the process of dopplerizing a transmitted signal $x(t)$, known analytically, proceeds as follows. The received signal is $y(t)=x\left(\mathcal{T}^{-1}(t)\right)$. The sampling operation at the receiver converts the received signal into the discrete set $\left\{y_{j}\right\}_{j=1,2,3, \ldots}$ where $y_{j}=y(j \Delta T)=x\left(\mathcal{T}^{-1}(j \Delta T)\right)=x\left(t_{j}\right)$ The term $\Delta T$ is the sample period in the receiver frame of reference, which is the reciprocal of the sample rate. Thus, at the $j$ th sampling instant at the receiver, the equation

$$
\mathcal{T}\left(t_{j}\right)=j \Delta T
$$

has to be solved for the root $t_{j}$. Once $t_{j}$ is known, the sample value is obtained analytically by direct evaluation [because $x(t)$ is known at every $t]$. This is done for $j=0,1,2, \ldots$ to construct the sampled, dopplerized received signal.

As an example, the motion-induced effects were examined in a simulation involving the path from the transmitter at the depth of $500 \mathrm{~m}$ at station T2300 (where the surface forcing was some of the most vigorous due to bad weather) to receiver $\mathbf{R}$ (see Fig. 1). This involves a southeast path with a range of approximately $1536 \mathrm{~km}$. The receiver is defined to be stationary.

Since a typical transmission protocol involved 1023-b $\mathrm{m}$-sequences [25] sent one after the other, without interruption, for many sequence repetitions, the simulated transmission was defined to be a train of waveforms starting at September 28, 2004 17:34:00Z and continuing for about $15 \mathrm{~h}$. The $m$-sequences had a carrier of $75 \mathrm{~Hz}$ and a period of $27.28 \mathrm{~s}$, for a total of 2016 complete sequences. This 15 -h signal was dopplerized and sampled according to the inverse mapping and the actual model-based trajectory at station T2300. This "received" signal was then blocked into 2016 segments, and each segment was processed with a standard pulse-compression algorithm. This yielded one dominant pulse per segment. The location of the pulse peak (usually between actual sample points) was estimated as the peak of a quadratic curve fit to the highest five samples, and the in-phase and quadrature $(I$ and $Q)$ components of the pulse peak were determined via linear interpolation. These (complex) interpolants for each $m$-sequence were then considered a (complex) time series.

An instructive presentation of the statistical properties of this time series is shown in Fig. 13. Here, the amplitudes of the $(I, Q)$ pairs have been normalized by the peak amplitude of an undopplerized waveform [i.e., the $(I, Q)$ constellation is now referenced to the unit circle]. In the absence of transmitter motion, all $(I, Q)$ points would occur at the same location on the circle. Instead, Doppler effects cause the $(I, Q)$ points to vary around the unit circle. Doppler compression or stretching also causes a mismatch in the pulse-compression correlator between

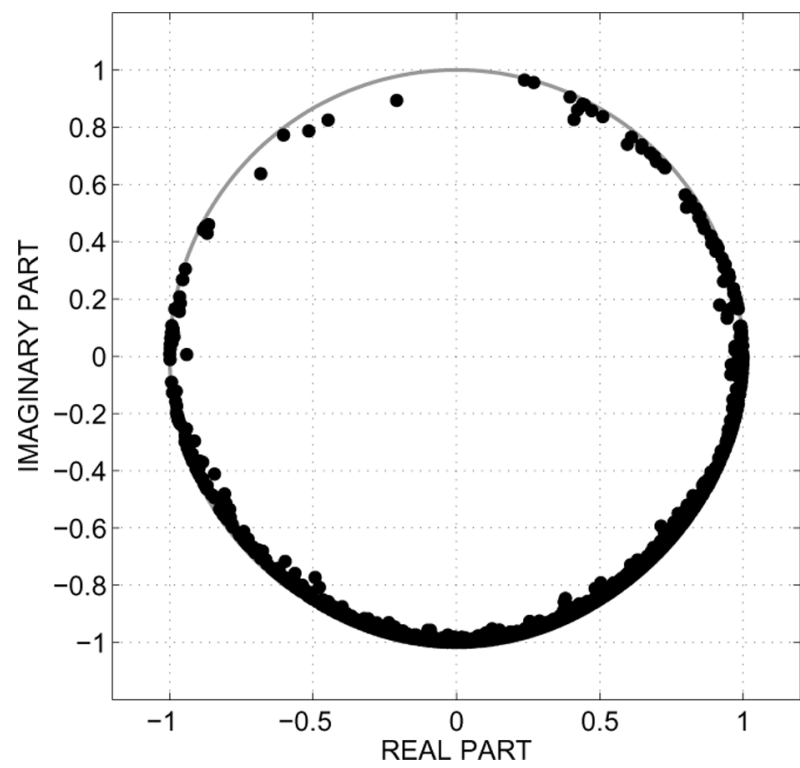

Fig. 13. Complex phase-space scatter plot of the in-phase (real) and quadrature (imaginary) components of the pulse peak, normalized by the undopplerized pulse amplitude. Simulation for station T2300, depth $500 \mathrm{~m}$, to receiver $\mathbf{R}$ for approximately $15 \mathrm{~h}$.

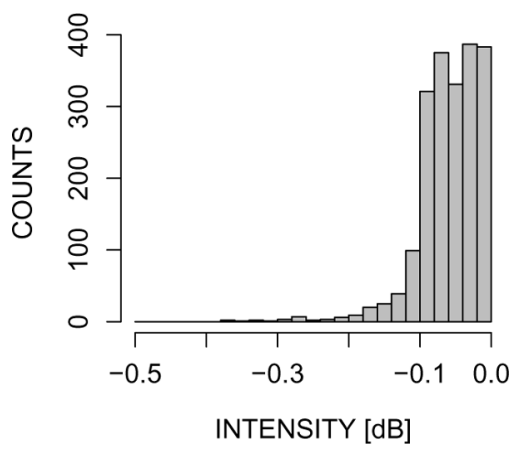

Fig. 14. Histogram of the peak intensities, normalized by the undopplerized pulse amplitude. Simulation for station T2300, depth $500 \mathrm{~m}$ to receiver $\mathbf{R}$, for approximately $15 \mathrm{~h}$.

the received signal and the replica, and this causes some $(I, Q)$ points to fall inside the unit circle.

Although this station had the worst weather, and consequently the most vigorous horizontal transmitter displacements, it can be seen from Fig. 13 that the induced Doppler did not redistribute the $(I, Q)$ points uniformly around the circle, which would represent a completely randomized signal. Rather, $(I, Q)$ points are grouped towards $-90^{\circ}$. Histograms of the peak intensity (Fig. 14) and the peak phase (Fig. 15) corroborate this observation. The peak amplitude fluctuated within about $0.1-0.2 \mathrm{~dB}$ of the ideal value, rarely more than $0.4 \mathrm{~dB}$. The peak phase was far from a phase-random process, with a dispersion of about 0.7 $\mathrm{rad}$ (the unit circle circumference is one wavelength, so this represents about $\lambda / 9)$ over a total span of about $4.9 \operatorname{rad}(4 \lambda / 5)$.

The temporal correlation properties of the intensity and phase are shown in Fig. 16 (normalized intensity) and Fig. 17 (phase). The intensity autocorrelation indicates that the intensity decorrelates after only a few $m$-sequences. The phase autocorrelation shows that phase remains correlated slightly longer. The 


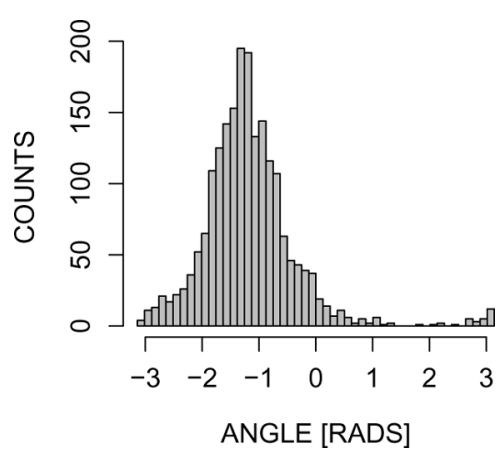

Fig. 15. Histogram of the peak phases (in radians). Simulation for station T2300, depth $500 \mathrm{~m}$ to receiver $\mathbf{R}$, for approximately $15 \mathrm{~h}$.

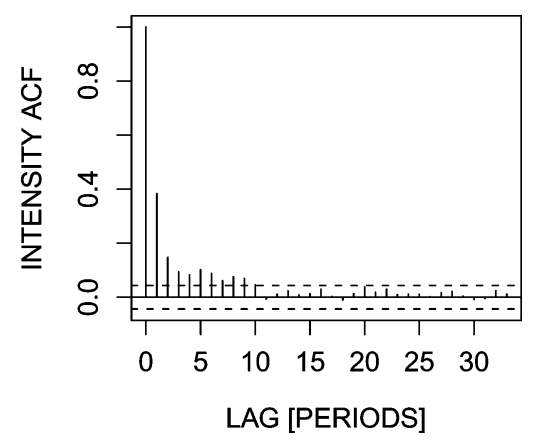

Fig. 16. Normalized intensity autocorrelation function. Lag is in units of individual $m$-sequence periods $(27.28 \mathrm{~s})$. Station T2300, depth $500 \mathrm{~m}$, to receiver R. (The dashed lines represent the $95 \%$ confidence interval assuming an uncorrelated series.)

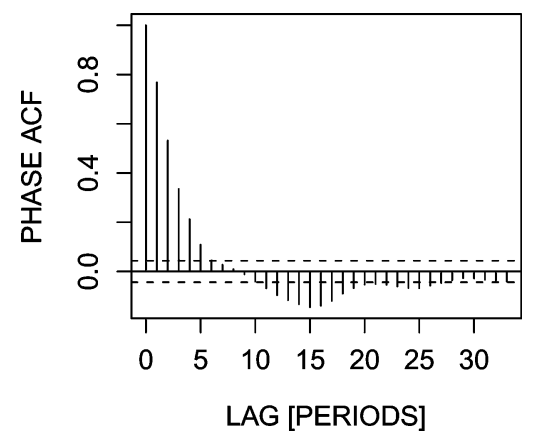

Fig. 17. Phase autocorrelation function. Lag is in units of individual $m$-sequence periods. Station T2300, depth $500 \mathrm{~m}$, to receiver $\mathbf{R}$. (The dashed lines represent the $95 \%$ confidence interval assuming an uncorrelated series.)

anticorrelation around lag 15 is likely to be related to the oscillations in the dynamic positioning system (see Fig. 7) with a period of about 5-10 min.

A common postprocessing operation is "coherent averaging," in which $J$ measurements are averaged together. This operation is intended to enhance features common in each measurement by diminishing additive noise power by $J$. In the simplest case, the data involve a deterministic "signal" component (which is identical in each successive measurement) and additive noise. In the present context, the signal is not deterministic but partially randomized as well, varying from measurement to measurement due to Doppler distortion caused by interplatform mo- tion. If this variation is slow, the signal component in $J$ successive measurements may have enough similarity that they will average "strongly," whereas the additive noise power decreases by $J$. This leads naturally to a consideration of the mean squared error (MSE) over $J$ samples. In the following, each "sample" is an entire $m$-sequence waveform.

Let the $j$ th waveform be

$$
\mathrm{z}_{j}=\mathrm{y}_{j}+\mathrm{n}_{j}
$$

where y is $L$-point dopplerized waveform, and $\mathrm{n}$ is $L$-point independent and identically distributed noise. Even though y is varying with time due to interplatform motion, both components are considered stationary over long time intervals. The signal power is $S=\left\langle\mathrm{y}^{T} \mathrm{y}\right\rangle$ and the noise power is $N=\left\langle\mathrm{n}^{T} \mathrm{n}\right\rangle$. In addition, the noise and the signal are assumed to be uncorrelated, so that $\left\langle\mathrm{y}^{T} \mathrm{n}\right\rangle=0$.

The "coherent" average is

$$
\mathrm{a}_{J}=\frac{1}{J} \sum_{j=1}^{J} \mathrm{z}_{j}=\frac{1}{J} \sum_{j=1}^{J} \mathrm{y}_{j}+\frac{1}{J} \sum_{j=1}^{J} \mathrm{n}_{j}=\mathrm{a}_{y, J}+\mathrm{a}_{n, J} .
$$

Let the $\mathrm{MSE}_{J}$ be the average amount, over $J$ consecutive waveforms, by which $\mathrm{a}_{y, J}$ deviates from the individual addends:

$$
\operatorname{MSE}_{J}=\left\langle\frac{1}{J} \sum_{j=1}^{J}\left|\mathrm{y}_{j}-\mathrm{a}_{y, J}\right|^{2}\right\rangle .
$$

The normalized mean squared error (NMSE) is

$$
\mathrm{NMSE}_{J}=\frac{\mathrm{MSE}_{J}}{S} \text {. }
$$

If the consecutive waveforms $\mathrm{y}_{j}$ are deterministic and therefore exactly alike, they are perfectly coherent and the $\mathrm{NMSE}_{J}$ is zero. On the other hand, if consecutive waveforms are completely randomized, the $\mathrm{NMSE}_{J}=1-1 / J$.

The NMSE was calculated for the series of dopplerized $m$-sequences described above, and a single realization of the NMSE curve is shown as a function of $J$ in Fig. 18. The effect of transmitter motion in this realization is to cause the NMSE to grow quickly, then level out beyond about 20 addends, paralleling the incoherent curve but always below it. This indicates partially coherent waveforms.

Processing gain is defined by $\mathrm{PG}_{J}=\mathrm{SNR}_{J} / \mathrm{SNR}_{1}$ where

$$
\mathrm{SNR}_{J}=\frac{\left\langle\left|\sum_{j=1}^{J} \mathrm{y}_{j}\right|^{2}\right\rangle}{\left\langle\left|\sum_{j=1}^{J} \mathrm{n}_{j}\right|^{2}\right\rangle}=\frac{J\left\langle\left|\mathrm{a}_{y, J}\right|^{2}\right\rangle}{N} .
$$

The SNR cannot be computed directly in the simulation above because there is no additive noise. However, the numerator is

$$
\left\langle\left|\sum_{j=1}^{J} \mathrm{y}_{j}\right|^{2}\right\rangle=\left\langle\sum_{j=1}^{J} \sum_{k=1}^{J} \mathrm{y}_{j}^{T} \mathrm{y}_{k}\right\rangle=\sum_{j=1}^{J} \sum_{k=1}^{J}\left\langle\mathrm{y}_{j}^{T} \mathrm{y}_{k}\right\rangle .
$$




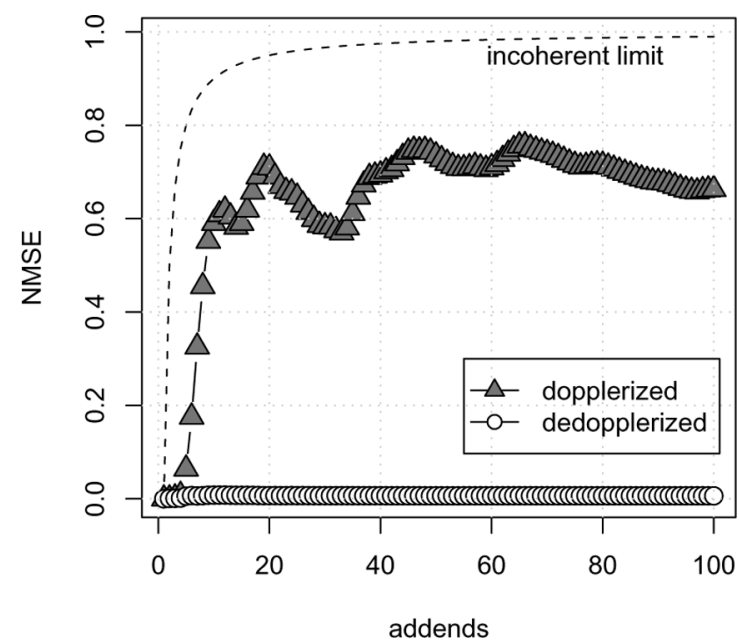

Fig. 18. NMSE of the average waveform against the individual waveforms. The "dopplerized" curve is for the simulation of station T2300, depth $500 \mathrm{~m}$ to receiver R. The "de-dopplerized" curve used an order 41 filter (see Section V). The coherent limit should have NMSE equal to zero, but the first de-dopplerized waveform has errors due to the filter startup transients: thereafter all waveforms are highly identical and the NMSE becomes independent of total addends.

Therefore, defining $\left\langle\mathrm{y}_{j}^{T} \mathrm{y}_{k}\right\rangle=S \rho_{j k}$, this becomes

$$
\left\langle\left|\sum_{j=1}^{J} y_{j}\right|^{2}\right\rangle=S \sum_{j=1}^{J} \sum_{k=1}^{J} \rho_{j k} .
$$

Note, however, that by expanding (7) in (8), one obtains

$$
\operatorname{NMSE}_{J}=1-\frac{1}{J^{2}} \sum_{j=1}^{J} \sum_{k=1}^{J} \rho_{j k}
$$

and, using (11), one can derive the relation between "processing gain" and NMSE

$$
\mathrm{PG}_{J}=J\left(1-\mathrm{NMSE}_{J}\right)
$$

The processing gain for perfectly coherent (i.e., deterministic) waveforms in additive noise goes as $J$, and for perfectly incoherent waveforms remains at 1 . A single realization of the processing gain curve calculated for the series of dopplerized $m$-sequences described above is shown as a function of $J$ in Fig. 19. The increasing departure with increasing total addends of the dopplerized curve from the perfectly coherent curve is further evidence of the generally "decohering" effect of transmitter motion on this algorithm. However, the fact that the dopplerized curve does not level off indicates that the influence is not completely randomized around the unit circle (Fig. 13).

In summary, the following general observations apply for the Doppler-induced signal variability during LOAPEX.

1) Doppler-induced intensity fluctuations are a few tenths of a decibel, infrequently half a decibel or more.

2) Doppler-induced travel time fluctuations (represented by the phase) are a few milliseconds, rarely more than $5 \mathrm{~ms}$.

3) Correlation in either peak intensity or phase persists for a few $m$-sequences.

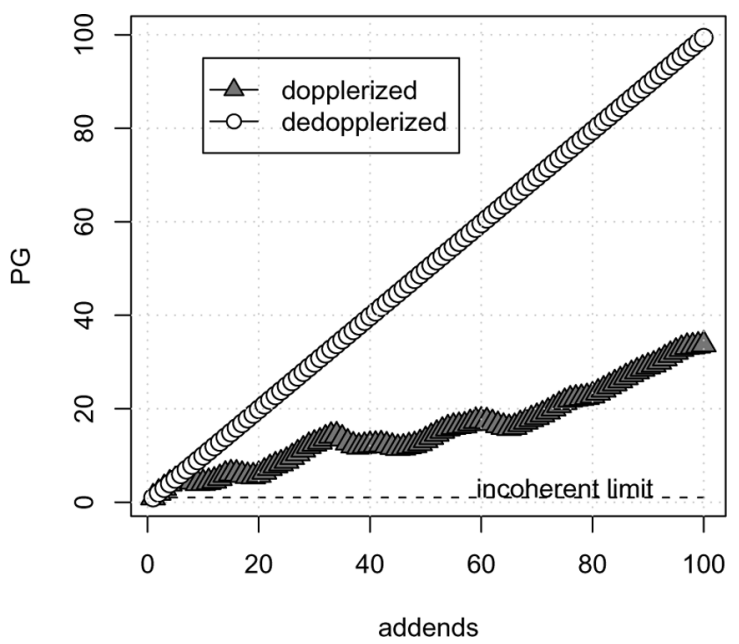

Fig. 19. Processing gain (PG) simulation. The "dopplerized" curve is for the simulation of station T2300, depth $500 \mathrm{~m}$ to receiver R. The "de-dopplerized" curve used an order 41 filter (see Section V).

4) Coherent averaging does not achieve the same processing gain against Doppler-induced fluctuations as against additive white Gaussian noise, due to MSE in consecutive waveforms.

A method for correcting the Doppler-induced time-base transformation is discussed in Section V.

\section{DOPPLER EFFECT REMOVAL}

Once one has the mapping $\mathcal{T}(t)$, one can construct a "dedopplerization" filter. De-dopplerization is defined here as the operation that converts a received signal $y\left(t^{\prime}\right)$ back to an estimate $\hat{x}\left(\mathcal{T}^{-1}\left(t^{\prime}\right)\right)$ of the transmitted signal $x(t)$. In the absence of any a priori information regarding the interplatform motion, the map $\mathcal{T}(t)$ must be estimated from the received data itself. This is typically the case with a noncooperative target, or when the accuracy of the tracking solutions is inadequate, or when trying to determine the Doppler effects of the ocean itself. however, the present context presumes highly accurate transmitter and receiver track solutions. Problems of this kind have arisen, for example, in the measurement at acoustical test ranges of radiated noise from vehicles moving past fixed receivers [26]. For a generalized problem involving arbitrary interplatform motion, the sampled de-dopplerized process is [27]

$$
\begin{aligned}
\hat{x}_{j} & =\hat{x}\left(t_{0}+j \Delta\right)=y\left(\mathcal{T}\left(t_{0}+j \Delta\right)\right) \\
& =\sum_{k=-\infty}^{\infty} y_{k} \operatorname{sinc} \sigma\left[\mathcal{T}\left(t_{0}+j \Delta\right)-\mathcal{T}\left(t_{0}\right)-k \Delta\right] \\
& \approx \sum_{k=-K}^{K} y_{k} \operatorname{sinc} \sigma\left[\mathcal{T}\left(t_{0}+j \Delta\right)-\mathcal{T}\left(t_{0}\right)-k \Delta\right]
\end{aligned}
$$

where $\sigma$ is the bandwidth of the signal and the mapping is assumed to be synchronized at some "epoch" $t_{0}$ in the transmitter frame of reference. $\Delta$ is the desired sample period in the final, de-dopplerized process. The summation must be truncated in implementation, hence the processor is implemented from (15) as an ordinary finite-impulse response (FIR) filter of order $2 K+$ 1 , with weight updates for every new sample $j$ output. 


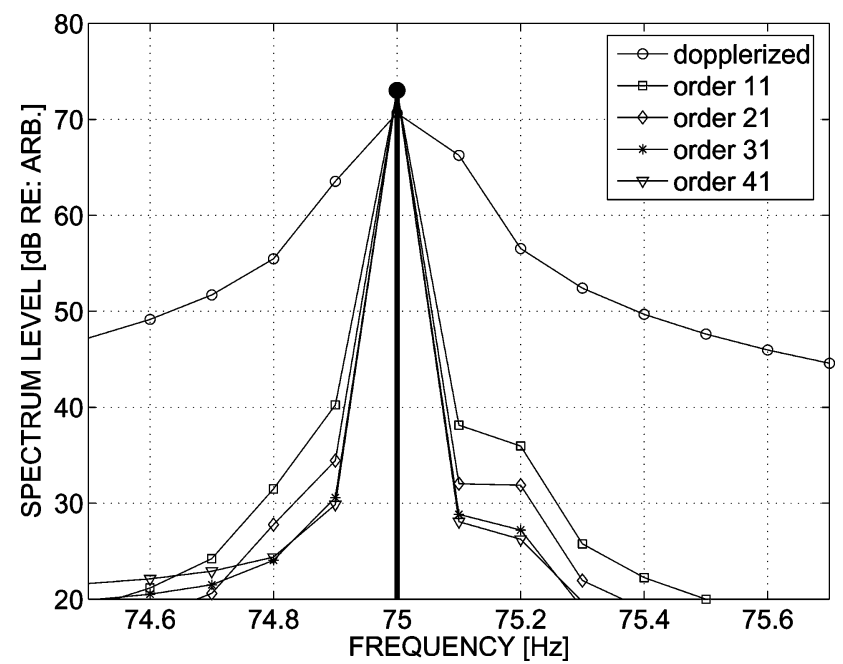

Fig. 20. Effect of filter order on reconstruction error. The spectral content of the undopplerized signal is represented by a single component at $75 \mathrm{~Hz}$ (heavy line).

\section{A. Doppler Broadening Simulation With a Sinusoid}

The effect of filter order choice can be quantified using a simple 75-Hz sinusoidal test signal. In the absence of time-base dilation, the spectral content of the signal is a single component at the carrier frequency. When the signal is dopplerized, spectral energy spreads to adjacent frequencies, an effect known as "Doppler broadening." This is shown in Fig. 20. (This example uses $50 \mathrm{~s}$ from a simplified scenario involving a transmitter revolving on a $10-\mathrm{m}$ radius circle with a 50 -s period and a receiver revolving on $100-\mathrm{m}$ radius circle with a 12 -h period.)

The spectral content of reconstructions using filter orders 11 , 21, 31, and 41 is also shown in Fig. 20. (To distinguish Doppler broadening from spectral leakage, one must use a uniform taper and a discrete Fourier transform window that contains an integral number of waveform periods.) All four filters provide considerable reduction in the error signal, with the predominant portion of the error signal energy concentrated within $\pm 0.5 \%$ of the carrier. Error signal rejection improves $3-5 \mathrm{~dB}$ from order 11 to 21 to 31, beyond which one obtains negligible additional improvement. According to Fig. 20, the practical maximum performance is reached at about order 41 .

\section{B. De-Dopplerizing the Dopplerized m-Sequence}

Application of an order 41 de-dopplerizing filter on the synthetic T2300 to receiver $\mathbf{R}$ data, generated in the simulation of Section IV-B, produced excellent results. The complex phasespace scatter plot is shown in Fig. 21: all $(I, Q)$ pairs are now clumped closely about $0^{\circ}$. The RMS phase error is $0.05 \mathrm{rad}$ $(\lambda / 121)$ over a span of $0.42 \mathrm{rad}(\lambda / 15)$, a decrease in phase error by more than an order of magnitude. The error from waveform to waveform is now negligible, and both the de-dopplerized NMSE and processing gain curves (Figs. 18 and 19) are almost indistinguishable from the coherent limit.

\section{SUMMARY}

An oceanographic cable dynamics model has been used to "track" the 3-D position versus time of an acoustical transmitter

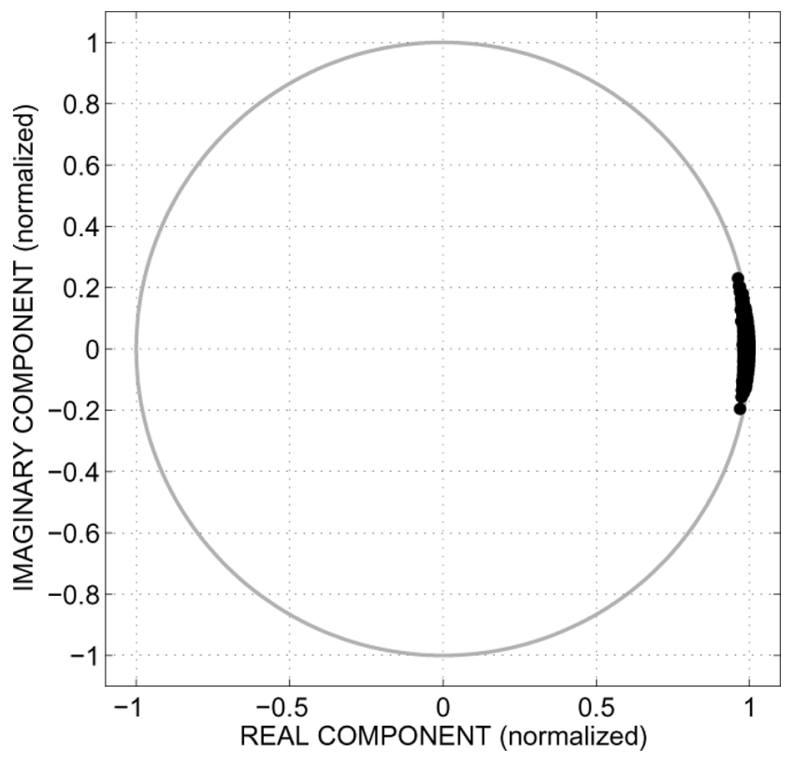

Fig. 21. Phase-space scatter plot after compensation.

suspended from a ship. The model inputs are mechanical parameters, fluid coefficients, and forcing functions given by 1) the motion of the top of the suspension cable, as measured by GPS, and 2) the currents along the cable to the depth of the transmitter, as measured by the ship's ADCP. For working sea states, the transmitter horizontal translations were of order $10 \mathrm{~m}$ or less.

Auxiliary measurements indicated that the cable model solution for the transmitter position was accurate to better than $\pm 2 \mathrm{~m}$ along either horizontal axis: this is better than $\lambda / 10$ at the nominal $75-\mathrm{Hz}$ transmission signal carrier frequency. Horizontal velocity estimates had an RMS error of about $0.05 \mathrm{~m} / \mathrm{s}$, or about $10 \%$ that of the RMS velocities themselves. These results suggest that this "tracking" approach is applicable to other precision low-frequency experiments.

The transmitter motion involves random accelerations, and therefore the corresponding Doppler effects are not simple to estimate. Direct simulation is used to show that the impact of interplatform motion depends on the desired data product. For example, measurements of deep intensity fades are essentially insensitive to Doppler-induced intensity variations. Coherent processors, particularly those spanning a considerable portion of the interplatform motion time scales (or longer), may require time-base correction. One correction solution, a simple time-varying de-dopplerization FIR filter, is shown to regain considerable signal fidelity, achieving nearly optimal coherent processing gain in simulation for 30-40 taps.

In general, the Doppler-induced degradation is not simply related to the platform motion(s) and generally will need to be investigated via simulation on a case-by-case basis.

\section{ACKNOWLEDGMENT}

The authors would like to thank J. I. Gobat (Applied Physics Laboratory, University of Washington, Seattle) who provided considerable guidance regarding the cable dynamics model; S. Liberatore (Woods Hole Oceanographic Institution, Woods Hole, MA) who loaned the two acoustical interrogators for the partial acoustical tracking system; and L. J. A. Colosi and J. Xu 
who operated the interrogators on the cruise. The C-Nav GPS system was rented from C\&C Technologies, Lafayette, LA. The crew of the $R / V$ Melville were very helpful, and in particular Computer Technician G. Davis, who provided the ADCP data, and Resident Technician B. Wilson, who kept the authors on their (own) schedule, oversaw all the rigging, supervised the scientists, and also demonstrated the one-handed method for tying a bowline.

\section{REFERENCES}

[1] R. B. Lindsay, "The story of acoustics," J. Acoust. Soc. Amer., vol. 39, pp. 629-644, 1966.

[2] A. Ayres and T. Calkins, "Acoustic position measurement systems," in Proc. OCEANS Conf., Los Angeles, CA, Oct. 17-19, 1977, vol. 2, pp. 45B-1-45B-4.

[3] W. H. Munk, P. F. Worcester, and C. Wunsch, Ocean Acoustic Tomography. Cambridge, U.K.: Cambridge Univ. Press, 1995, pp. 209-209.

[4] R. C. Spindel, R. P. Porter, W. M. Marquet, and J. L. Durham, "A high resolution pulse-Doppler underwater acoustic navigation system," IEEE J. Ocean. Eng., vol. OE-1, no. 1, pp. 6-13, Sep. 1976.

[5] B. R. Steinberg, Principles of Aperture and Array System Design. New York: Wiley, 1976, pp. 301-329.

[6] B. M. Howe, J. A. Mercer, R. C. Spindel, and P. F. Worcester, "Accurate positioning for moving ship tomography," in Proc. OCEANS Conf., Seattle, WA, 1989, vol. 3, pp. 880-886.

[7] W. H. Munk, R. C. Spindel, A. Baggeroer, and T. G. Birdsall, "The Heard Island feasibility test," J. Acoust. Soc. Amer., vol. 96, no. 4, pp. 2330-2342, 1991.

[8] M. A. Dzieciuch, W. H. Munk, and A. Forbes, "Interpretation of GPS offsets from a steady course," J. Atmos. Ocean. Technol., vol. 9, pp. 866-892, 1992.

[9] P. F. Worcester, B. D. Cornuelle, M. A. Dzieciuch, W. H. Munk, B. M. Howe, J. A. Mercer, R. C. Spindel, J. A. Colosi, K. Metzger, T. G. Birdsall, and A. B. Baggeroer, "A test of basin-scale acoustic thermometry using a large-aperture vertical array at $3250 \mathrm{~km}$ range in the eastern North Pacific Ocean," J. Acoust. Soc. Amer., vol. 105, no. 6, pp. 3185-3201, 1999.

[10] P. F. Worcester, B. M. Howe, J. A. Mercer, M. A. Dzieciuch, T. G. Birdsall, K. Metzger, and R. C. Spindel, "A comparison of long-range acoustic propagation at the ultra-low $(28 \mathrm{~Hz})$ and very low $(84 \mathrm{~Hz})$ frequencies," in Proc. U.S.-Russian Workshop Exp. Underwater Acoust., Nyzhny Novgorod, Russia, Nov. 18-19, 1999, pp. 93-104.

[11] J. A. Mercer, R. K. Andrew, B. M. Howe, and J. A. Colosi, "Cruise report: Long-range Ocean Acoustic Propagation EXperiment (LOAPEX)," Appl. Phys. Lab., Univ. Washington, Seattle, WA, Tech. Rep. APL-UW TR 0501, 2005.

[12] J. A. Mercer, J. A. Colosi, B. M. Howe, M. A. Dzieciuch, R. Stephen, and P. F. Worcester, "LOAPEX: The Long-range Ocean Acoustic Propagation EXperiment," J. Ocean. Eng., vol. 34, no. 1, pp. 1-11, Jan. 2009.

[13] B. M. Howe, S. G. Anderson, A. Baggeroer, J. A. Colosi, K. R. Hardy, D. Horwitt, F. W. Karig, S. Leach, J. A. Mercer, K. Metzger, L. O. Olson, D. A. Peckham, D. A. Reddaway, R. R. Ryan, K. von der Heydt, R. P. Stein, J. D. Watson, S. L. Weslander, and P. F. Worcester, "Instrumentation for the acoustic thermometry of ocean climate (ATOC) prototype Pacific Ocean network," in Proc. OCEANS Conf., 1995, vol. 3 , pp. $1483-1500$.

[14] J. I. Gobat and M. A. Grosenbaugh, "WHOI cable v2.0: Time domain numerical simulation of moored and towed oceanographic systems," Woods Hole Oceanogr. Inst., Woods Hole, MA, Tech. Rep. WHOI2000-08, 2000.

[15] A. A. Tjavaras, "Dynamics of highly extended cables," Ph.D. dissertation, Dept. Ocean. Eng., Massachusetts Inst. Technol., Cambridge, MA, 1996.

[16] J. I. Gobat and M. A. Grosenbaugh, "Application of the generalized- $\alpha$ method to the time integration of the cable dynamics equations," Comput. Methods Appl. Mech. Eng., vol. 190, pp. 4817-4829, 2002.

[17] J. Chung and G. M. Hulbert, "A time integration algorithm for structural dynamics with improved numerical dissipation," J. Appl. Mech., vol. 60, pp. 371-375, 1993.
[18] W. H. Press, S. A. Teukolsky, W. T. Vetterling, and B. P. Flannery, Numerical Recipes in C, 2nd ed. Cambridge, U.K.: Cambridge Univ. Press, 1992.

[19] A. H. Sherman, "Algorithms for sparse Gaussian elimination with partial pivoting," ACM T. Math. Softw., vol. 4, pp. 330-338, 1978.

[20] D. R. Yoerger, M. A. Grosenbaugh, M. S. Triantafyllou, and J. J. Burgess, "Drag forces and flow-induced vibrations of a long vertical tow cable-Part I: Steady-state towing conditions," J. Offshore Mech. Arctic Eng., vol. 113, pp. 117-127, 1991.

[21] M. A. Grosenbaugh, D. R. Yoerger, F. S. Hover, and M. S. Triantafyllou, "Drag forces and flow-induced vibrations of a long vertical tow cable-Part II: Unsteady tow conditions," J. Offshore Mech. Arctic Eng., vol. 113, pp. 199-204, 1991.

[22] C. M. Alexander, "The complex vibrations and implied drag of a long oceanographic wire in cross-flow," Ocean Eng., vol. 8, no. 4, pp. 379-406, 1981.

[23] M. R. Zarnetske, "Long-range Ocean Acoustic Propagation Experiment (LOAPEX): Preliminary analysis of transmitter motion and tidal signals," M.S. thesis, School Oceanogr., Univ. Washington, Seattle, WA, 2005.

[24] A. R. Gondeck, "Doppler time mapping," J. Acoust. Soc. Amer., vol. 73 , no. 5 , pp. $1863-1864,1983$.

[25] T. G. Birdsall and K. Metzger Jr., "M-sequence signal tutorial," Commun. Signal Process. Lab., Electr. Eng. Comput. Sci. Dept., Univ. Michigan, Ann Arbor, MI, Naval Oceanogr. Office Presentation, 1988.

[26] J. Hay, "Improvement of the imaging of moving acoustic sources by the knowledge of their motion," in Proc IEEE Conf. Acoust. Signal Process., 1981, vol. 3, pp. 1247-1252.

[27] S. A. L. Glegg, "The de-dopplerization of acoustic signals using digital filters," J. Sound Vib., vol. 116, no. 2, pp. 384-387, 1987.

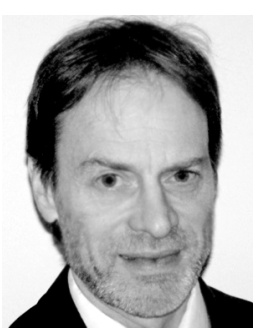

Rex K. Andrew (M'88-S'91-M'98) received the B.S. degree in physics and the M.S.E.E. degree from the University of Washington, Seattle, in 1981 and 1987, respectively, and the Ph.D.E.E. degree from the University of Victoria, Victoria, BC, Canada, in 1997.

From 1987 to 1991 , he was with the Naval Undersea Warfare Engineering Station, Keyport, WA, where he led an effort to develop large-aperture discrete-element over-the-side acoustical measurement systems. From 1997 to 1998, he was with the Department of Radiology, University of Washington, where he developed diagnostic ultrasound video codecs using 3-D wavelets. From 1997 to 1999, he developed 2-D image registration and alignment algorithms with iOptics, Inc. He joined the Applied Physics Laboratory, University of Washington, in 1999, where he is currently a Senior Engineer. His major research interests are statistical signal and array processing, wave propagation in random media, oceanic ambient noise, and acoustical oceanography.

Dr. Andrew is a member of the IEEE Signal Processing Society and the Acoustical Society of America.

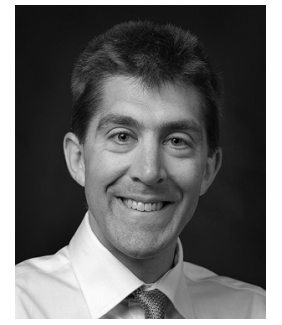

Michael R. Zarnetske received the B.S. degree in ocean engineering from Florida Institute of Technology, Melbourne, in 1997, the M.S. degree in ocean engineering from the University of Rhode Island, Kingston, in 1999, and the M.S. degree in physical oceanography from the University of Washington, Seattle, in 2005.

$\mathrm{He}$ has worked at the Naval Undersea Warfare Center (NUWC) Division Newport, Newport, RI, since 2008. He previously worked at Raytheon Integrated Defense Systems and Ultra Electronics Ocean Systems, where he was involved in sonar system design, testing, and integration. At NUWC, he is involved with a number of projects focusing on the development and testing of piezoelectric and single crystal sonar transducers.

Mr. Zarnetske is a member of the Acoustical Society of America and the National Defense Industrial Association. 


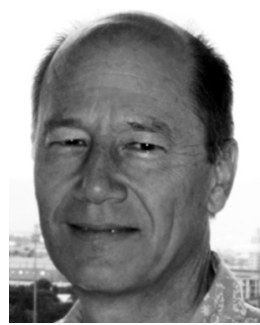

Bruce M. Howe (M'07) received the B.S. degree in mechanical engineering and the M.S. degree in engineering science from Stanford University, Stanford, CA, in 1978 and the Ph.D. degree in oceanography from the Scripps Institution of Oceanography, University of California, San Diego, in 1986

From 1986 to 2008, he worked at the Applied Physics Laboratory and is currently a Professor at the Ocean and Resources Engineering Department, University of Hawaii at Manoa, Honolulu. While at Stanford University, he developed laser Doppler velocimetry (LDV) instrumentation for air-sea interaction experiments. From 1979 to 1981, he was a Research Associate with the Institut für Hydromechanik, Universität Karlsruhe, Germany, working on LDVs for use in the atmospheric boundary layer. While at the Scripps Institution and since then, he has worked on many ocean acoustic tomography projects, including moving ship tomography, acoustic thermometry of ocean climate (ATOC) and the North Pacific Acoustic Laboratory (NPAL). He helped establish ongoing Ocean Observatories efforts, and is working on fixed infrastructure (e.g., cable power systems and moorings), mobile platforms (e.g., gliders as navigation/communications nodes and acoustic receivers), and hybrids (e.g., moored vertical profilers). A goal is to integrate acoustic systems in ocean observing to provide navigation, communications, timing, and science applications.

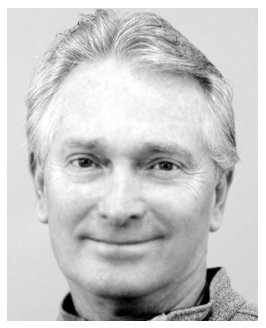

James A. Mercer received the B.S. degree in physics and the Ph.D. degree in geophysics from the University of Washington, Seattle, in 1968 and 1983, respectively.

He is a Principal Physicist at the Applied Physics Laboratory and a Research Professor at the Department of Earth and Space Sciences, University of Washington, Seattle.

Dr. Mercer is an Associate Editor of the U.S. Navy Journal of Underwater Acoustics. 